\title{
The Effect of Anti-L-Selectin (Aselizumab) on the Posttraumatic Inflammatory Response in Multiply Traumatized Patients
}

\author{
Andreas Seekamp ${ }^{1}$, Martijn van Griensven ${ }^{2}$, Christian Rusu², Jochem König ${ }^{3}$, \\ Jasmin Khan-Boluki ${ }^{4}$, Heinz Redl ${ }^{5}$
}

\begin{abstract}
Purpose: This prospective phase II parallel-group, double-blind, randomized, placebo-controlled clinical trial was meant to evaluate the effect of a humanized monoclonal anti-L-selectin antibody (aselizumab) on the posttraumatic inflammatory response in severely injured patients.

Patients and Methods: To be eligible for the study, patients had to have sustained a trauma due to a blunt or penetrating injury, which involved at least two organ systems according to the Abbreviated Injury Scale (AIS) resulting in a total Injury Severity Score (ISS) of $\geq 25$. Included patients $(n=84)$ received either placebo or $0.5 \mathrm{mg} / \mathrm{kg}, 1.0 \mathrm{mg} / \mathrm{kg}$, or $2.0 \mathrm{mg} / \mathrm{kg}$ of aselizumab within $6 \mathrm{~h}$ of the traumatic event. Parameters representing the posttraumatic inflammatory response were monitored for 10 days, patients' general records were monitored for 14 days and discontinuously until day 42 . Results: The incidence of multiple organ failure (MOF) defined as a median value of the total Goris MOF score of $\geq 5$ on $\geq 2$ consecutive days within the first 14 days was not significantly different for the placebo and the $0.5-\mathrm{mg} / \mathrm{kg}, 1.0-\mathrm{mg} / \mathrm{kg}$, and $2.0-\mathrm{mg} / \mathrm{kg}$ groups. The cytokine interleukin-(IL-) 6 and IL-10 serum levels as well as neutrophil elastase serum concentrations revealed the well-described posttraumatic acute response which resolved from day 3 after trauma in all four study groups.
\end{abstract}

Also complement factor $\mathrm{C}_{3} \mathrm{a}$ and procalcitonin followed the same pattern. There were no statistically significant differences between placebo and patients having received anti-L-selectin at different dosages.

Conclusion: For all of the measured variables, IL-6, IL-10, elastase, $\mathrm{C}_{3}$ a and procalcitonin, there were only a few trends but no significant differences between all study groups indicating that the effect of the administered anti-L-selectin antibody on the posttraumatic inflammatory response was similar to that of placebo.

\section{Key Words}

Multiple trauma $\cdot$ Organ failure $\cdot$ L-selectin .

Neutrophil adhesion · Inflammation · Clinical trial

Eur J Trauma 2005;31:557-67

DOI 10.1007/s00068-005-1066-4

\section{Introduction}

One principal event in the pathophysiology of multiple organ failure (MOF) is the excessive uncontrolled activation of endogenous inflammatory mediators and immunocompetent cells, in particular, neutrophil granulocytes. Among the inflammatory mediators especially the cytokines interleukin-(IL-)6 and IL-10 have been recurrently identified to play a major role. IL-6 serum

\footnotetext{
'Department of Traumatology, Saarland University Medical School, Homburg/Saar, Germany,

2 Department of Traumatology, Hanover Medical School, Hanover, Germany,

${ }^{3}$ Department of Medical Statistics, Epidemiology and Medical Informatics, Saarland University Medical School, Homburg/Saar, Germany,

${ }^{4}$ Scil Biomedicals GmbH, Martinsried, Germany,

${ }^{5}$ Ludwig Boltzmann Institute for Experimental and Clinical Traumatology, Vienna, Austria.
}

Received: December 14, 2004; revision accepted: March 1, 2005. 
levels increase in patients suffering from MOF and therefore, IL-6 is considered to be an inflammatory agonist $[1,2]$. By contrast, IL-10 seems to be an inflammatory antagonist, as IL-10 administration reduced mortality in different experimental models of sepsis and MOF [3, 4]. It was also reported that IL-10 deficiency may contribute to the development of MOF [5] and the increase of the IL-6/IL-10 ratio indicated poor outcome [6]. In recent years also the acute-phase protein procalcitonin (PCT) has been shown in experimental as well as clinical studies to be a significant indicator of posttraumatic complications [7-9], although its pathophysiological role is not totally identified yet. The combined analysis of IL-6, PCT and complement factor C3a revealed a high reliability to distinguish between sepsis and systemic inflammatory response syndrome (SIRS) in medical intensive care patients [10].

Activated neutrophils interfere with the vascular endothelium, which finally results in capillary leakage through proteinases such as elastase [11] and other mediators [12]. The crucial step in the sequence of events leading to endothelial injury seems to be the interaction between the neutrophils and the endothelial cell which is mediated by adhesion molecules. These are expressed on cell surfaces either already under basal conditions or upon activation through cytokines like IL-6 [13] or factors of the complement cascade [14].

The process of neutrophil adherence occurs mainly in two steps requiring different adhesion molecules. The first step of neutrophil-endothelial cell interaction (rolling) is mediated by the selectin family of adhesion molecules; the second step is firm adhesion mediated by neutrophil integrins (sticking); definite adhesion is followed by diapedesis of activated neutrophils through the endothelial cell layer, leading to interstitial neutrophil migration $[15,16]$.

In order to block this process, the selectin family of adhesion molecules are one potential target, as any kind of selectin antagonism may interfere with one of the first steps of neutrophil adhesion. Two of the selectins (E- and P-selectin) are mainly localized on endothelial cells and can be activated through different mechanisms [13], whereas L-selectin is mainly expressed by circulating leukocytes and is involved in the localization of lymphocytes, monocytes and neutrophils to activated endothelium at the site of inflammation [17]. Therefore, it was expected that blocking of L-selectin may be more effective than antagonizing one of the other selectins, which may even be expressed in organ-specific patterns.
In experimental studies blocking of L-selectin attenuated the remote lung injury after infrarenal ischemia/reperfusion injury [18] without increasing the risk of infection, improved survival in a baboon trauma model [19], and diminished pulmonary microvascular permeability in burn and smoke injury [20]. As part of a clinical phase II trial in multiply traumatized patients using a monoclonal antibody (aselizumab) directed against the adhesion molecule L-selectin [21], this study evaluated the effect of that specific antibody on relevant mediators of the posttraumatic inflammatory response (IL-6, IL-10, complement factor C3a, neutrophil elastase, and PCT), that had been measured in parallel to the clinical trial.

\section{Patients and Methods \\ General Aspects}

The underlying clinical trial was designed as a parallel-group, double-blind, randomized, placebo-controlled study involving multiply traumatized patients at risk of MOF, and was performed in 14 trauma centers in Central Europe. The study was initiated and sponsored by Scil Biomedicals GmbH (Martinsried, Germany) and monitored by Covance Clinical and Periapproval Services Ltd. (Leeds, UK). This prospective study was conducted in accordance with the current version of the Declaration of Helsinki. In addition, it was conducted in accordance with the laws, regulations, scientific and ethical standards of research of each country and Good Clinical Practice (GCP). The study was approved by each local ethics committee. Informed consent was required in written form prior to study entry and was either given by the patient him-/herself or by the next of kin. In the latter case the patient had to sign the informed consent as soon as he/ she was able to give consent.

\section{Study Population}

Inclusion criteria. Male and female patients, aged between 18 and 64 years, had to have sustained a trauma due to a blunt or penetrating injury, which involved at least two organ systems according to the Abbreviated Injury Scale (AIS) [22], been able to receive the antibody within $6 \mathrm{~h}$ of the traumatic event and been expected to survive at least $48 \mathrm{~h}$ from the time of drug administration. The time of the traumatic event was defined as the time of resuscitation at the scene. The trauma must have resulted in a total Injury Severity Score (ISS) of $\geq 25$ or a single organ system AIS score of 4 in one of at least two different injured organ systems. Women of childbearing potential had to have a negative pregnancy 
test. Patients or their legally acceptable representatives had to provide informed consent.

Exclusion criteria. Patients were excluded when violating one of the inclusion criteria and were also not included when having sustained a devastating head injury (Glasgow Coma Scale $\leq 8$ ), received an investigational drug within 30 days before study entry, previously received monoclonal antibody therapy, had a known chronic disease such as renal failure or liver disease unsuitable for study entry, or had previously received cardiopulmonary resuscitation as a result of the traumatic injury suffered at the scene, in transit or in the emergency department.

After patient eligibility had been confirmed, all patients were assigned to one of the three aselizumab groups or placebo group according to the randomization $(3: 1)$ list.

\section{Study Drug Administration}

Patients were randomly allocated to receive either aselizumab (HuDregg-55), a recombinant humanized monoclonal antibody (IgG4) directed against the lectin domain of the adhesion molecule L-selectin [23], at dosages of $0.5 \mathrm{mg} / \mathrm{kg}, 1.0 \mathrm{mg} / \mathrm{kg}$, and $2.0 \mathrm{mg} / \mathrm{kg}$ or placebo (composition of vehicle: dibasic potassium phosphate, monobasic potassium phosphate, L-arginine, sucrose, polysorbate 80 , phosphoric acid). For each patient the correct volume of study medication to be administered to that patient was calculated according to the patient's body weight. All doses were administered as a once-only dose via a central venous line. Diluent was added to make up a final volume of $50 \mathrm{~cm}^{3}$ which then was delivered using a syringe-driven pump over a 15 -min period. Study drug was administered as soon as feasible after the traumatic event and not more than $6 \mathrm{~h}$ after the documented time of the traumatic event.

Following i.v. infusion, maximum serum levels of aselizumab generally occurred at the first sampling time point after the start of infusion (30 $\mathrm{min})$ at each dose level. This was followed by a plateau in aselizumab serum levels up to $48 \mathrm{~h}$. The elimination half-life of aselizumab was generally similar at each dose with mean values ranging from 115 to $211 \mathrm{~h}$. Neutrophil binding site saturation was measured by fluorescence-activated cell sorting (FACS) methodology. Whole-blood samples were drawn from patients and fixed until further processing. The saturation of neutrophil binding sites was determined by FACS utilizing DREG56, which binds to a different CD62L locus and served as an indi- cator of CD62L density on neutrophils, and a specific anti-hIgG4 antibody, which had previously been shown to bind to the study drug. The percentage of neutrophil CD62L saturation was thus calculated from the ratio of DREG56 antibody binding and anti-hIgG4 binding. The maximum saturation of neutrophil binding sites was high $(>89 \%)$ in all patients receiving aselizumab and similar across the $0.5-\mathrm{mg} / \mathrm{kg}$ to $2-\mathrm{mg} / \mathrm{kg}$ dose range. This high level of saturation was maintained between $30 \mathrm{~min}$ and $24 \mathrm{~h}$ after infusion. Thereafter, the saturation fell slowly and in a dose-dependent manner.

\section{Protocol and Parameters}

Patients included in the study were monitored once per day continuously for the first 14 days and discontinuously at days 20,30, and 42 . At day 180 a phone call visit finished the study. Besides parameters that had to be taken only once initially to confirm the patient eligibility, further parameters were monitored such as physical examination, vital signs, therapeutic interventions, MOF score data and blood samples were taken for arterial blood gases, hematology, clinical chemistry and special analysis.

Out of the mediators considered to reflect the inflammatory response after trauma a variation of five parameters was chosen and monitored only for the first 10 days after trauma: IL-6, IL-10, neutrophil elastase, C3a, and PCT. All of these parameters were analyzed in one central laboratory using commercially available test kits: human IL-6 ELISA (sensitivity 1.4 pg/ml, Cat. No. BMS213/2CE, Bender MedSystems Vienna, Austria), human IL-10 ELISA (sensitivity $0.8 \mathrm{pg} / \mathrm{ml}$, Cat. No. BMS215/2, Bender MedSystems Vienna, Austria), PMN-elastase ELISA (sensitivity $2.0 \mathrm{pg} / \mathrm{ml}$, Cat. No. MKEL1, Milenia Biotec, Bad Nauheim, Germany), C3a ELISA (sensitivity $3.7 \mathrm{ng} / \mathrm{ml}$, Cat. No. A015, Biomedica, Vienna, Austria), PCT-LUMItest ${ }^{\circledR}$ (sensitivity 0.08 ng/ml, BRAHMS, Henningsdorf, Germany).

\section{Evaluation of Organ Dysfunction}

Data for the assessment of pulmonary, cardiac, renal, hepatic, hematologic, gastrointestinal, and central nervous system (CNS) function were recorded to define the presence or absence of specific organ dysfunction, employing the Goris MOF scale in its original [24] and revised edition [25] between day 1 and day 14 . The daily total MOF score was the sum of the individual organ system scores. The daily individual organ scores ranged from 0 to 2 and the median total MOF scores ranged from 0 to 14 for all 
seven organ systems. The incidence of MOF was defined as a total MOF score $\geq 5$ on $\geq 2$ consecutive days recorded between day 1 and day 14 .

\section{Statistics}

All basic data including demographics, injury severity, morbidity, mortality, and MOF score data, were analyzed and compared between all treatment groups and the placebo group. If data for a particular variable were normally distributed, then treatment comparisons were made using an analysis of variance with pairwise post-hoc comparisons according to Student-Newman-Keuls. All biochemical data, non-normally distributed, were transferred into $\log$ values (base 10) prior to analysis of variance, in order to achieve approximate normal distribution and homogeneity of variance. MOF score data were analyzed using a rank test for nonparametric data (Kruskal-Wallis test). The level of statistical significance was considered at $\mathrm{p}<0.05$, all tests comparing two conditions are two-sided. For analysis of correlations the Pearson method was employed. Data are presented graphically as mean \pm 2 SEM on the log scale.

\section{Results}

\section{Study Population}

According to the inclusion criteria a total of 84 patients were recruited, 23 were randomized to receive aselizumab at $0.5 \mathrm{mg} / \mathrm{kg}, 21$ to receive aselizumab at $1 \mathrm{mg} / \mathrm{kg}$, 20 to receive aselizumab at $2 \mathrm{mg} / \mathrm{kg}$, and 20 patients to receive placebo. Demographic data as well as the severity of injuries were well balanced between all groups (Table 1). However, there were significantly more males than females in the study, but the number of females was higher in the placebo group compared with the aselizumab groups. All the patients were Caucasian. In all groups the major trauma always involved the chest and the extremities at first.

\section{Morbidity and Mortality}

Indicators of overall morbidity (time spent on ventilator, length of stay in intensive care unit [ICU], total du- ration of hospitalization, and cumulative fluid balance) were not significantly different between all four groups (Table 2).

Overall, twelve patients died and were subsequently withdrawn as serious adverse events from the study. The incidence of death was highest in the placebo group $(\mathrm{n}=5)$, followed by the aselizumab $0.5-\mathrm{mg} / \mathrm{kg}$ group $(\mathrm{n}=4)$, the $1.0-\mathrm{mg} / \mathrm{kg}$ group $(\mathrm{n}=2)$, and the $2.0-\mathrm{mg} / \mathrm{kg}$ group $(\mathrm{n}=1)$. Overall mean survival time was 11.5 days (9.2 days in the placebo group, 13.3 days in the $0.5-\mathrm{mg} /$ $\mathrm{kg}, 9.5$ days in the $1.0-\mathrm{mg} / \mathrm{kg}$, and 19 days in the $2.0-\mathrm{mg} /$ $\mathrm{kg}$ group) without any significant difference between all groups. None of the deaths was considered to be directly related to the study drug but rather to injury severity and subsequent MOF without any significant differences between all study groups (Table 2). In detail, seven patients died due to MOF or ARDS (adult respiratory distress syndrome), three patients died due to their traumatic brain injury, and two patients suffered from respiratory failure caused by direct pulmonary injury.

\section{Multiple Organ Failure Score}

As all inflammatory parameters are monitored only for 10 days, the mean total MOF score is also shown only 
Table 2. Indicators of overall morbidity and mortality. ARDS: adult respiratory distress syndrome; ICU: intensive care unit; MOF: multiple organ failure; NS: not significant; SD: standard deviation; TBI: traumatic brain injury.

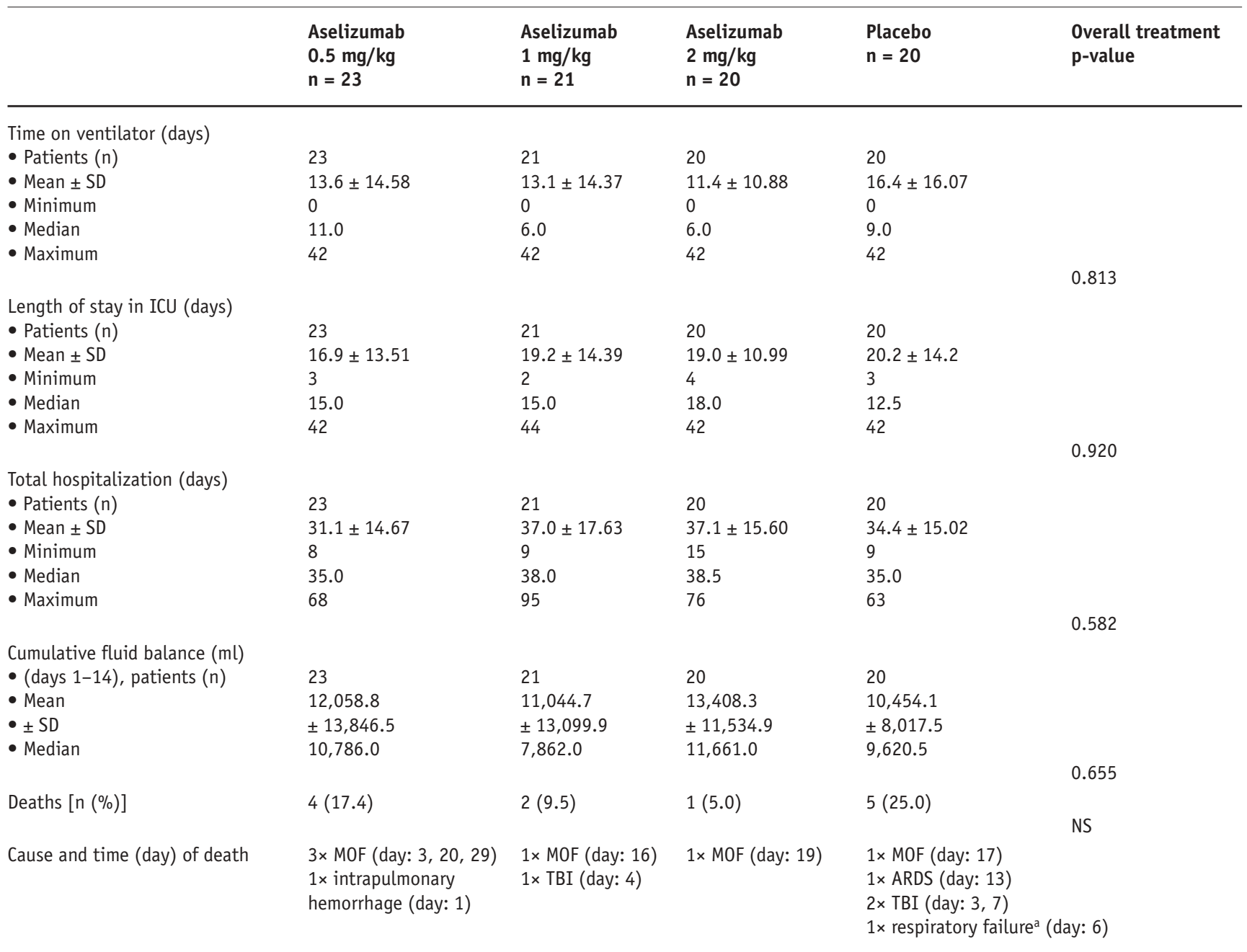

a due to airway injury

for this time period in this context. The mean total MOF scores according to the Goris MOF score [24] during day 1 and day 10 are presented in Figure 1 according to treatment groups. The study population presented the majority of patients in each study group with median total MOF scores in the range of $0-3$. The overall difference between the four groups as well as the pairwise treatment comparison were found to be statistically nonsignificant. The revised Goris MOF score [25] also provided results (not displayed) that were consistent with the standard Goris method.

\section{Parameters of the Inflammatory Response}

Within the first $6 \mathrm{~h}$ IL- 6 serum levels of the placebo group were measured at the maximal level of the 10-day period, subsequently followed by a slow but constant decline. The anti-L-selectin antibody could not demonstrate any significant change in the IL-6 serum levels at any time in any of the administered dosages. The IL-6 serum levels are almost running in parallel over the three study groups and the placebo group (Figure 2).

About the same pattern was demonstrated by the IL-10 serum levels. Again, a high peak was evident initially after trauma followed by a more rapid decline until day 3 and a plateau until day 10 . No statistically significant differences could be noted between all groups (Figure 3). Also the IL-6/IL-10 ratio was calculated and subjected to analysis of covariance and regression without any evidence of statistically significant differences between all groups (data not shown in detail). 


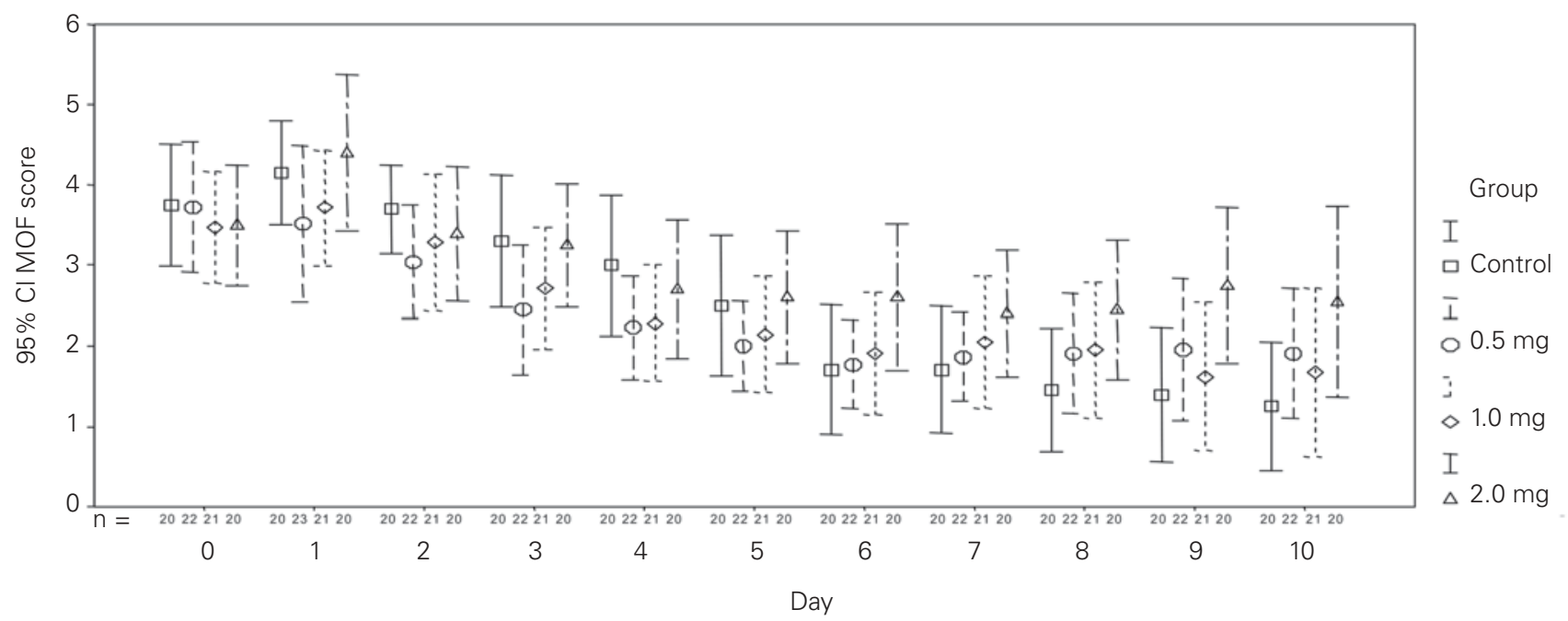

Figure 1. MOF score [24] for all study groups. Cl: confidence interval.

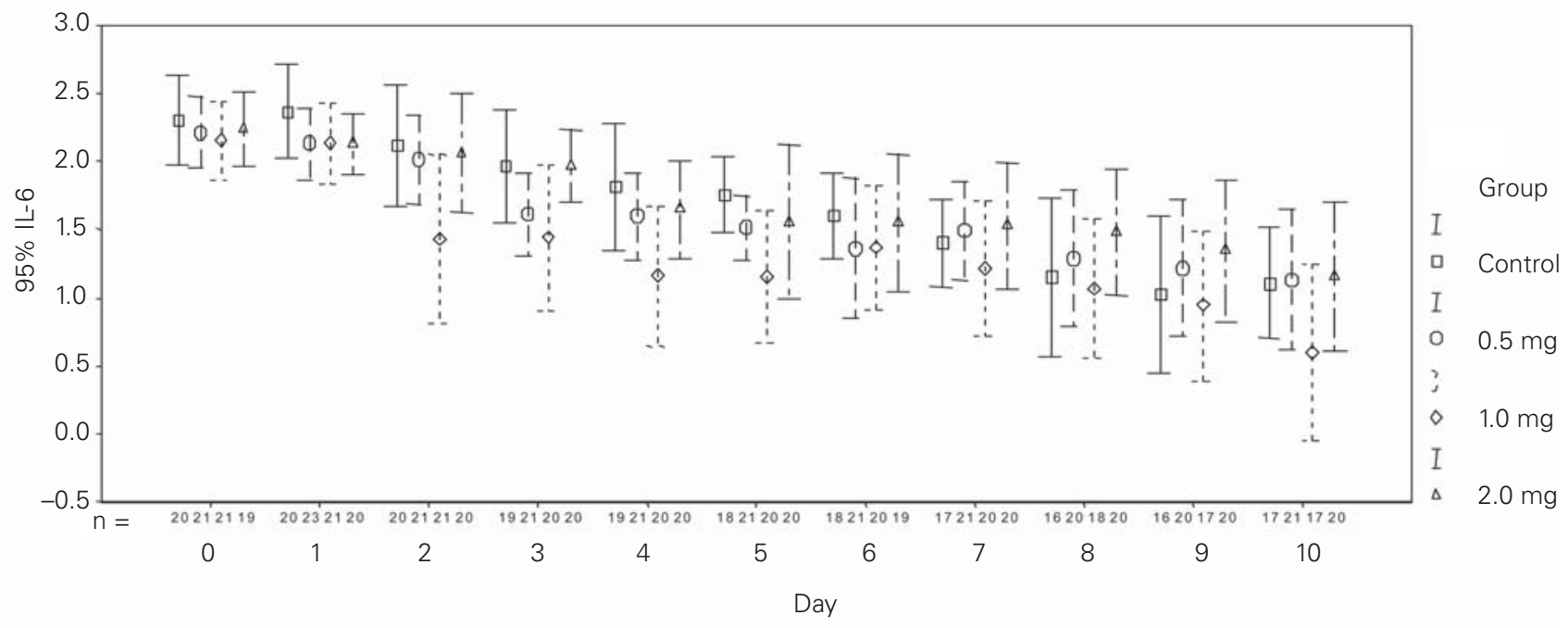

Figure 2. Serum interleukin-6 (pg/ml), log scale. $\mathrm{Cl}$ : confidence interval.

Complement factor $\mathrm{C} 3 \mathrm{a}$ serum levels were low in all groups initially after trauma and at day 1 . Again, no significant influence by either concentration of the anti-L-selectin was noted. After the initial depth C3a levels slightly increased reaching a plateau at day 8 after trauma (Figure 4).

Neutrophil elastase serum levels revealed a maximum on day 1 after trauma in all groups except the $0.5-\mathrm{mg} / \mathrm{kg}$ aselizumab group. Patients of this group started out with lower elastase serum levels initially and reached a maximum on day 3. Despite this obvious difference various statistical analyses have given no evidence of significance (Figure 5). Over the study period elastase serum levels of all patients declined continu- ously with minor but not significant differences between all groups.

PCT serum levels were noted low on day 0 and day 1 in all groups, followed by a sudden rise on day 2 which occurred in all three study groups and the placebo group. Subsequently, values declined again down to a level similar to that of day 1 . Interestingly, PCT measurements of the $1.0-\mathrm{mg} / \mathrm{kg}$ aselizumab group were running parallel to the other three groups but on a constant lower level. Significant differences were not evident at any time point (Figure 6).

In view of the insignificant results regarding the measured mediators it had to be examined if injury severity and the change in multiple organ function were at all re- 


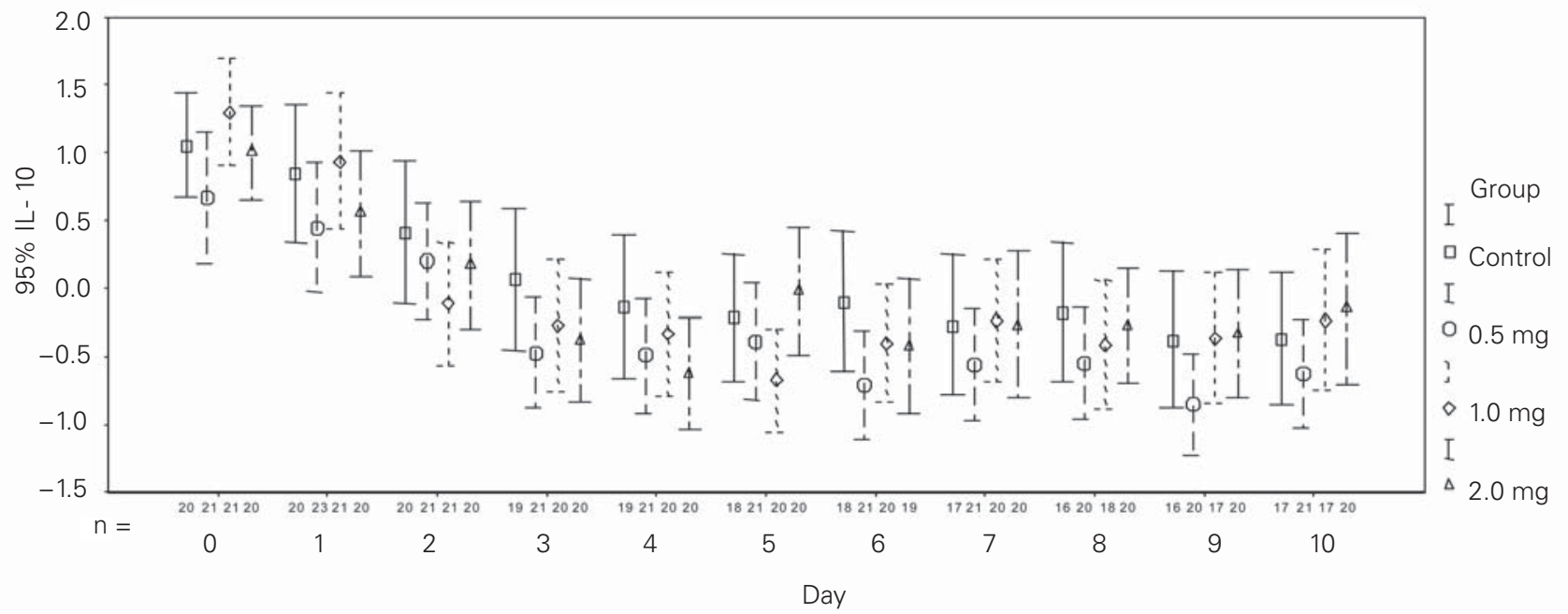

Figure 3. Serum interleukin-10 (pg/ml), log scale. Cl: confidence interval.

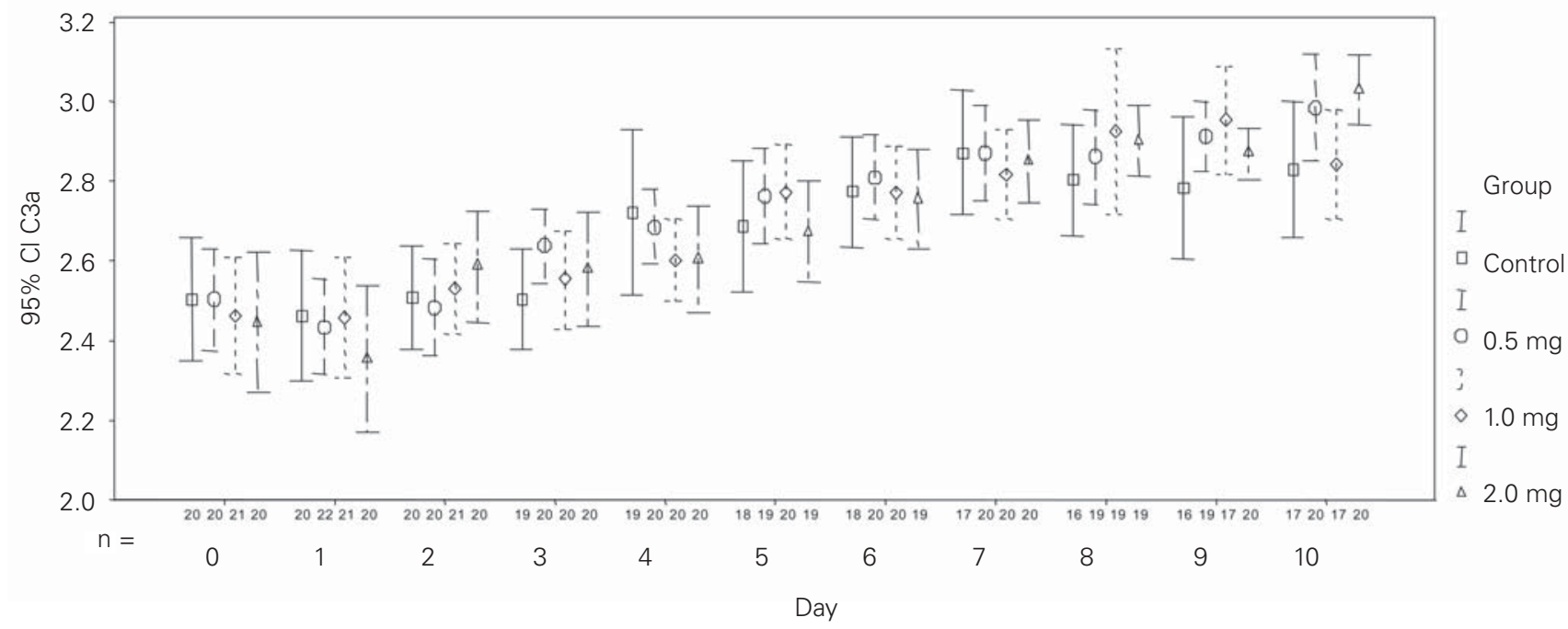

Figure 4. Serum complement factor $\mathrm{C}_{3}$ ( $\left.\mathrm{ng} / \mathrm{ml}\right)$, log scale. $\mathrm{Cl}$ : confidence interval.

flected by any of the measured parameters. Therefore, different correlations were analyzed cumulatively over all study patients. Injury severity in fact correlated with the degree of organ function (Goris MOF score; $p<0.02$ ). For all inflammatory parameters their correlation with the corresponding daily MOF score was also analyzed. For all parameters significant (Figures 7 and 8) correlations with the MOF score could be noted, especially IL- 6 and PCT most consistently correlated with the MOF score over the observation time. Yet, for none of the parameters a critical threshold could be identified to predict total outcome.

\section{Discussion}

The pathophysiology of MOF after major trauma is characterized by an inflammatory process involving dif- ferent mediators and the neutrophil granulocyte as one of the key cells. The crucial step of events leading to neutrophil migration and increased vascular permeability subsequently is the interaction between neutrophils and endothelial cells, which is mediated by adhesion molecules expressed on the surface of either cell type [26]. The adhesion molecule L-selectin, expressed on neutrophils, was identified to mediate the initial phase of transient adhesion [27]. The cell expression of L-selectin as well as the serum concentration of soluble L-selectin change significantly after trauma and may distinguish between patients developing organ failure and those who do not [28]. Therefore, L-selectin was supposed to be a potential target for immunomodulatory therapy [29]. Experimental studies [18, 20] strongly sup- 


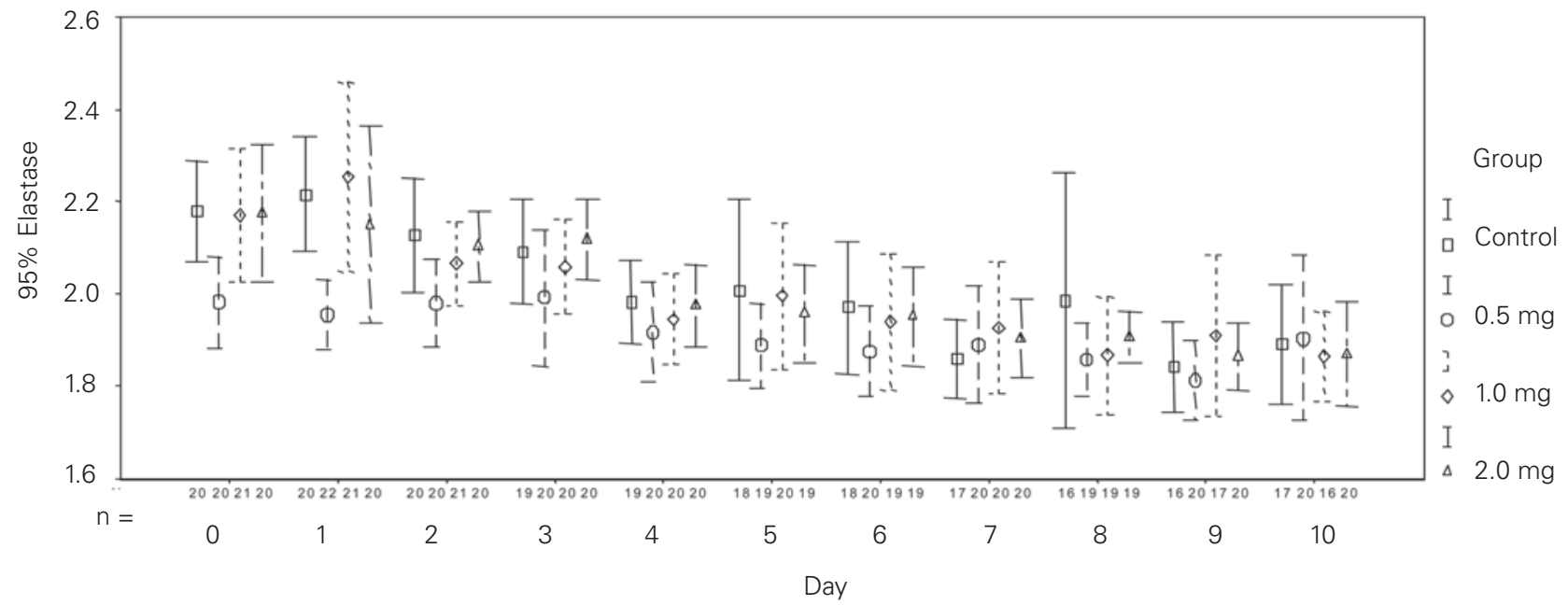

Figure 5. Neutrophil elastase serum levels (pg/ml), log scale. Cl: confidence interval.

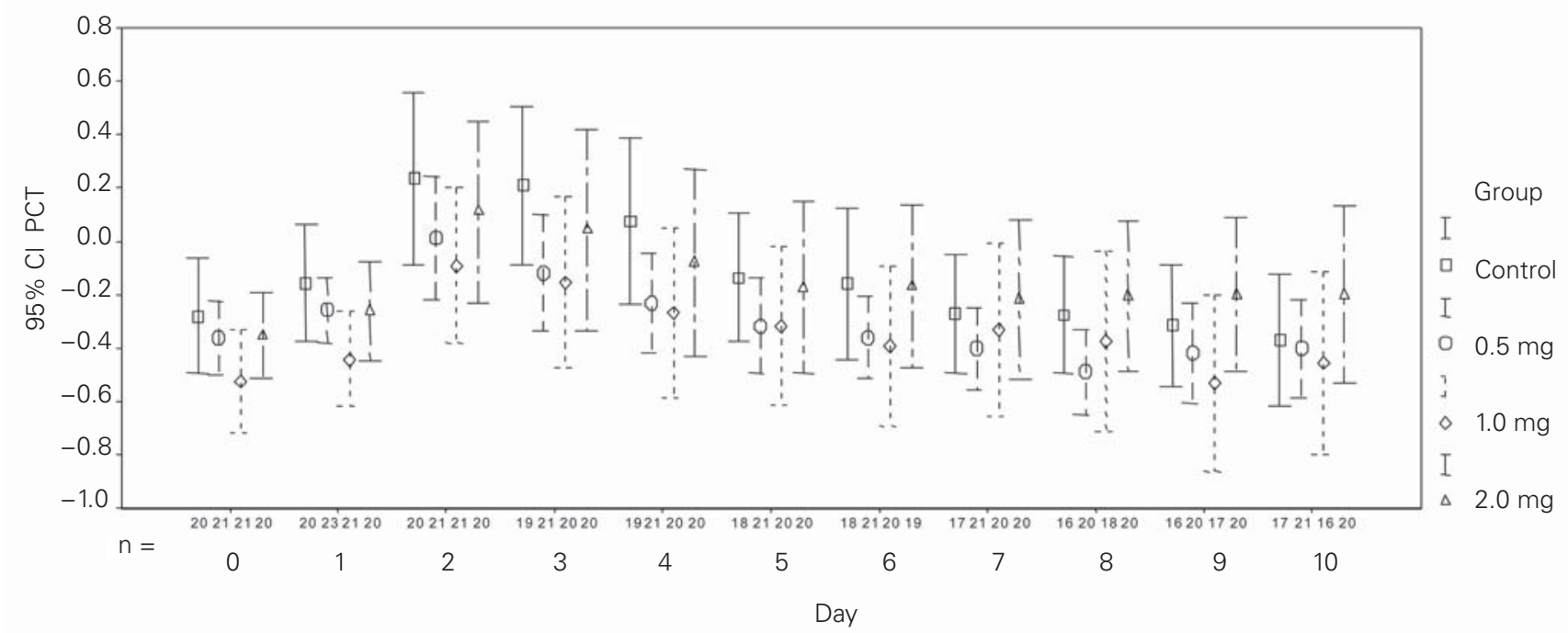

Figure 6. Serum procalcitonin (ng/ml), log scale. Cl: confidence interval.

ported this hypothesis and in fact the same antibody (aselizumab) used in this study significantly reduced mortality in a hemorrhagic-traumatic shock model in baboons [19]. Considering the encouraging experimental data, this study was designed to evaluate the effect of L-selectin blockade on inflammatory parameters in trauma patients at risk of MOF.

Yet, in fact none of the measured parameters (IL-6, IL-10, C3a, neutrophil elastase, and PCT) revealed a significant difference between placebo and the different aselizumab groups. By contrast, all parameters considered to reflect the posttraumatic inflammatory response presented the well-known acute-phase response. Except for complement factor $\mathrm{C} 3 \mathrm{a}$, which was depleted after trauma, IL-6, as well as IL-10, elastase and PCT increased within the first 3 days after trauma, meaning that all study patients have sustained an adequate injury which has resulted in a posttraumatic response of the immune system. However, this response was not influenced by aselizumab at different dosages. According to previous reports cytokines and complement factors are potential activators of neutrophils on one hand $[13,14]$, but on the other hand activated neutrophils are also a potential source of cytokines [12]. Therefore, it could not be expected to totally avoid IL-6 and IL-10 as well as $\mathrm{C} 3 \mathrm{a}$ release by anti-L-selectin but at least cytokine serum levels could have been diminished to some degree in case there was a significant effect of anti-L-selectin. As even neutrophil elastase was not different between placebo and aselizumab groups, it seems to be 


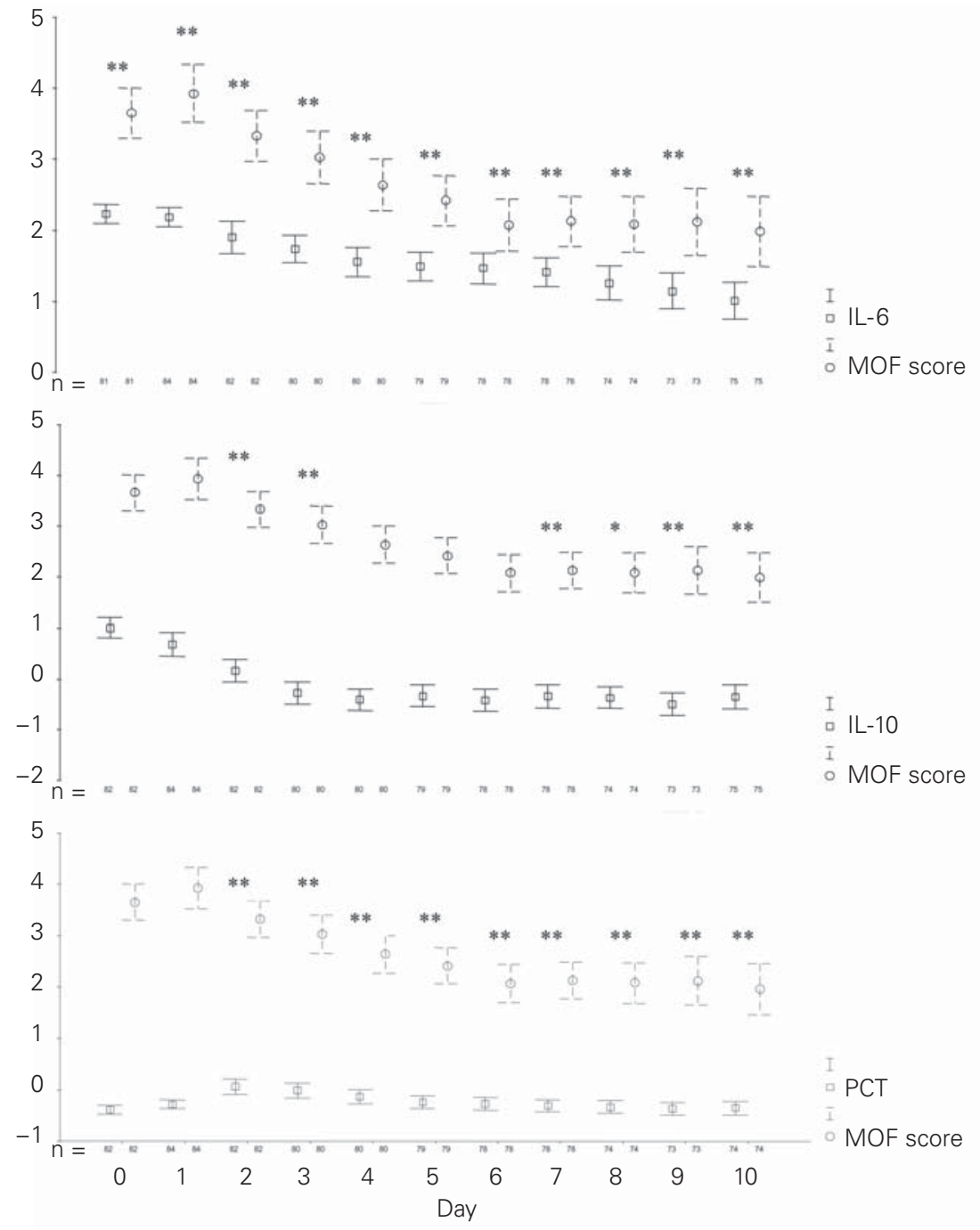

Figure 7. Correlation between IL-6, IL-10, procalcitonin (PCT) and MOF score. The $y$-axis holds a decimal scale for the MOF score and represents a log scale for the correlated parameter. Correlation was calculated by pairwise analysis of all available data of one parameter for each day. ${ }^{*} p<0.05,{ }^{* *} p<0.01$ indicates a significant correlation according to the Pearson method.

relations between cytokine serum levels as well as PCT and the Goris MOF score were also evident in this study.

The clinical course of all study patients as represented by the median total MOF score, the overall morbidity and mortality were not influenced by anti-L-selectin in this setting. There were only few trends in some of the parameters but no statistically significant differences between all study groups [30].

Different mechanisms can be discussed why anti-L-selectin had no effect on the inflammatory response after trauma. Gender distribution, which is different between groups, may have contributed to the nonsignificant effect of aselizumab on the inflammatory parameters. The percentage of females is twice as high in the placebo group compared to any other aselizumab group. It has been reported that females are at lower risk of posttraumatic complications, which may be related to different sex hormone levels [31]. Therefore, the placebo group of this study may have responded to trauma by a low inflammatory reaction just because of the relatively high number of females. The time of drug administration, initially after trauma, is currently believed to be the best period of action for drugs to inhibit neutrophil migration. However, a longer time period,

evident that no neutrophil activity related to adhesion and subsequent mediator release was inhibited by anti-L-selectin.

Various coefficients were also calculated for some of the monitored parameters in correlation to injury severity and organ dysfunction, as previous studies have identified the IL-6/IL-10 ratio as a predictive indicator of MOF. The imbalance of these two cytokine in favor of IL-6 was reported to contribute to organ failure [5, 6]. PCT, C3a and IL-6 have been recommended as discriminating parameters for sepsis and SIRS [10]. Independently of anti-L-selectin administration, significant cor- e.g., several days, may also be more beneficial. During the clinical trial aselizumab clearly presented dose-dependent serum levels and was proven to sufficiently interact with the neutrophil L-selectin (89\% saturation of neutrophil L-selectin) in the phase I and II study [30]. However, it has to be considered that the remaining $10 \%$ of active L-selectin, not covered by the antibody, may be sufficient to mediate neutrophil migration initiating organ dysfunction. There is experimental evidence that $>90 \% \mathrm{~L}$-selecting neutralization is required to achieve any significant attenuation of neutrophil adhesion [32]. For the antibody used in this study cross-linking with sur- 

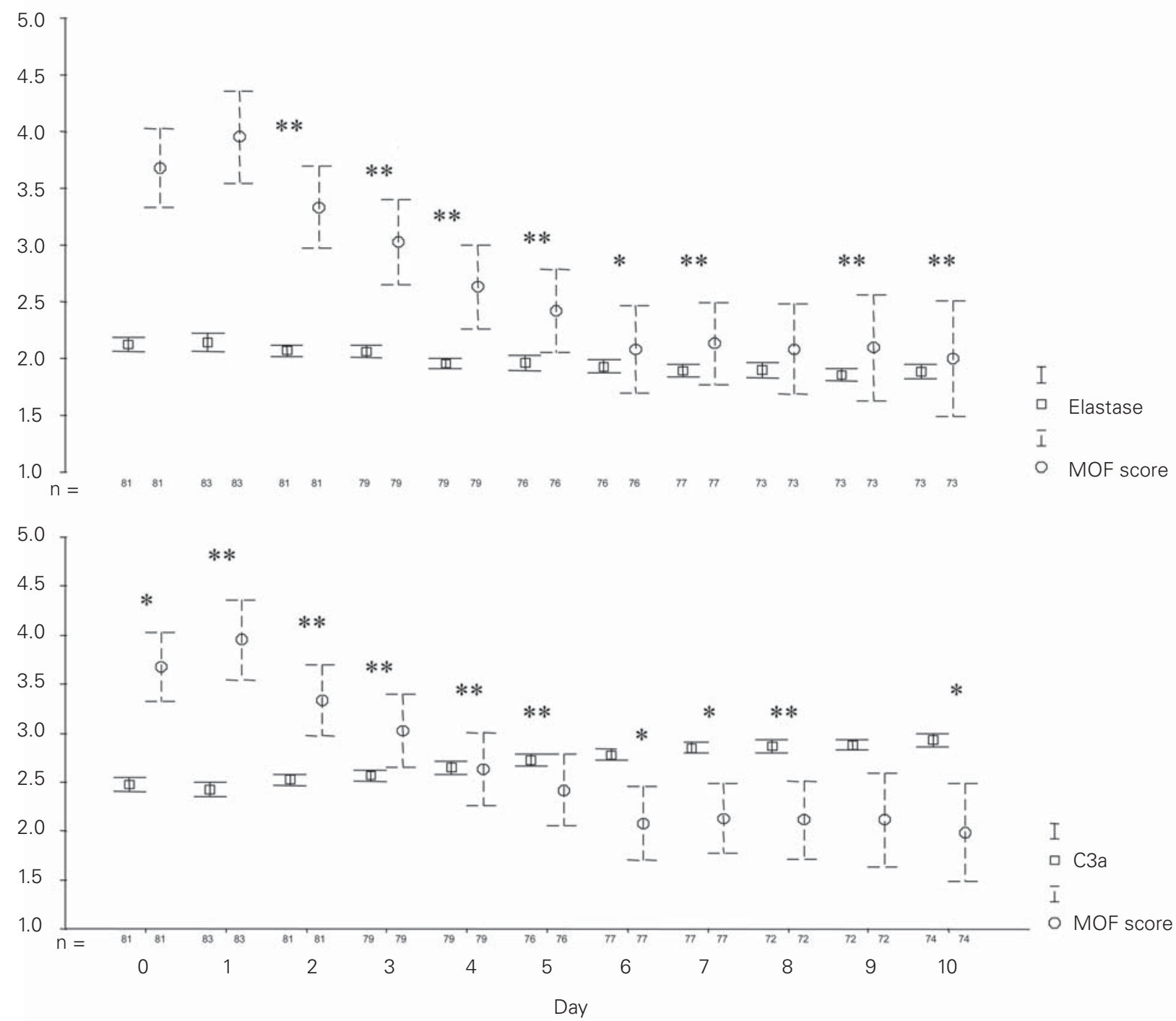

Figure 8. Correlation between neutrophil elastase, complement factor C3a and MOF score. Again, the $y$-axis holds a decimal scale for the MOF score and represents a log scale for the correlated parameter. Correlation was calculated by pairwise analysis of all available data of one parameter for each day. ${ }^{*} p<0.05,{ }^{* *} p<0.01$ indicates a significant correlation according to the Pearson method.

face L-selectin was described as one mechanism for neutrophil activation and L-selecting shedding [33]. Shedding of surface L-selectin may result in diminished neutrophil binding sites for the antibody and, in addition, some amount of the antibody may bind to the soluble L-selectin, as shown in vitro [28]. Thus, even higher concentrations of aselizumab may not necessarily result in a $100 \%$ saturation of neutrophil L-selectin. As always in the case of antagonizing one mechanism other physiological pathways besides the interaction of neutrophils and endothelial cells via the L-selectin adhesion molecule may also have come into play and thus may have overwhelmed the effect of L-selectin inhibition [34].

\section{Conclusion}

All study patients have presented the typical acute inflammatory reaction in response to the sustained severe trauma. However, this response was not influenced by blocking the L-selectin adhesion molecule using the specific monoclonal anti-L-selectin antibody aselizum$\mathrm{ab}$ at different dosages.

\section{References}

1. Seekamp A, Jochum M, Ziegler M, et al. Cytokines and adhesion molecules in elective and accidental trauma related ischemia reperfusion. J Trauma 1998;44:874-82. 
2. Hensler T, Sauerland S, Bouillon B, et al. Association between injury pattern of patients with multiple injuries and circulating levels of soluble tumor necrosis factor receptors, interleukin- 6 and interleukin-10, and polymorphonuclear neutrophil elastase. J Trauma 2002;52:662-709.

3. Latifi SO, O'Riordan MA, Levine AD. Interleukin-10 controls the onset of irreversible septic shock. Infect Immun 2002;70:4441-6.

4. Jansen MJ, Hendriks T, de Man BM, et al. Interleukin 10 mitigates the development of the zymosan-induced multiple organ dysfunction syndrome in mice. Cytokine 1999;11:713-21.

5. Loisa P, Rinne T, Laine S, et al. Anti-inflammatory cytokine response and the development of multiple organ failure in severe sepsis. Acta Anaesthesiol Scand 2003;47:319-25.

6. Taniguchi T, Koido Y, Aiboshi J, et al. Change in the ratio of interleukin- 6 to interleukin-10 predicts a poor outcome in patients with systemic inflammatory response syndrome. Crit Care Med 1999;27:1262-4.

7. Dehne MG, Sablotzki A, Hoffmann A, et al. Alterations of acute phase reaction and cytokine production in patients following severe burn injury. Burns 2002;28:535-42.

8. Redl H, Schlag G, Togel E, et al. Procalcitonin release patterns in a baboon model of trauma and sepsis: relationship to cytokines and neopterin. Crit Care Med 2000;28:3659-63.

9. Wanner GA, Keel M, Steckholzer U, et al. Relationship between procalcitonin plasma levels and severity of injury, sepsis, organ failure, and mortality in injured patients. Crit Care Med 2000; 28:950-7.

10. Selberg O, Hecker H, Martin M, et al. Discrimination of sepsis and systemic inflammatory response syndrome by determination of circulating plasma concentrations of procalcitonin, protein complement 3a, and interleukin-6. Crit Care Med 2000;28:2793-8.

11. Kinoshita $M$, Ono $S$, Mochizuki H. Neutrophils mediate acute lung injury in rabbits: role of neutrophil elastase. Eur Surg Res 2000; 32:337-46.

12. Zallen $\mathrm{G}$, Moore EE, Johnson JL, et al. Circulating postinjury neutrophils are primed for the release of proinflammatory cytokines. J Trauma 1999;46:42-8.

13. Dayal SD, Hasko G, Lu O, et al. Trauma/hemorrhagic shock mesenteric lymph upregulates adhesion molecule expression and IL-6 production in human umbilical vein endothelial cells. Shock 2002;17:491-5.

14. Foreman KE, Vaporciyan AA, Bonish BK, et al. C5a induced expression of P-selectin in endothelial cells.J Clin Invest 1994;94:1147-52.

15. Hakkert BC, Kuijpers TW, Leeuwenberg JF, et al. Neutrophil and monocyte adherence to and migration across monolayers of cytokine-activated endothelial cells: the contribution of CD18, ELAM-1, and VLA-4. Blood 1991;78:2721-6.

16. Luscinskas FW, Cybulsky MI, Kiely JM, et al. Cytokine-activated human endothelial monolayers support enhanced neutrophil transmigration via a mechanism involving both endothelial-leukocyte adhesion molecule-1 and intercellular adhesion molecule-1. J Immunol 1991;146:1617-25.

17. Kerner T, Ahlers O, Spielmann S, et al. L-selectin in trauma patients: a marker for organ dysfunction and outcome? Eur J Clin Invest 1999;29:1077-86.

18. Seekamp A, Regel G, Rother K, et al. The effect of anti-L-selectin (EL-246) on remote lung injury after infrarenal ischemia/reperfusion. Shock 1997;7:447-54.
19. Schlag G, Redl H, Till GO, et al. Anti-L-selectin antibody treatment of hemorrhagic-traumatic shock in baboons. Crit Care Med 1999;27:2050-3.

20. Katahira J, Murakami K, Schmalstieg FC, et al. Role of anti-L-selectin antibody in burn and smoke inhalation injury in sheep. Am J Physiol Lung Cell Mol Physiol 2002;283:L1043-50.

21. Co MS, Landolfi NF, Nagy JO, et al. Properties and pharmacokinetics of two humanized antibodies specific for L-selectin. Immunotechnol 1999;4:253-66.

22. Civil ID, Schwab CW. The Abbreviated Injury Scale, 1985 revision: a condensed chart for clinical use. J Trauma 1988;28:87-90.

23. Fu H, Berg EL, Tsurushita N. Fine mapping of the epitopes of humanized anti-L-selectin monoclonal antibodies HuDreg-55 and HuDreg-200. Immunol Lett 1997;59:71-8.

24. Goris RJ, te Boekhorst TP, Nuytinck JK, et al. Multiple-organ failure. Generalized autodestructive inflammation. Arch Surg 1985;120:1109-15.

25. Lefering R, Goris RJ, van Nieuwenhoven EJ, et al. Revision of the multiple organ failure score. Langenbecks Arch Surg 2002;387:14-20.

26. Eppihimer MJ, Granger DN. Ischemia/reperfusion-induced leukocyte-endothelial interactions in post capillary venules. Shock 1997;8:16-26.

27. Tedder TF, Steeber DA, Chen A, et al. The selectins: vascular adhesion molecules. FASEB J 1995;9:866-9.

28. Seekamp A, van Griensven M, Hildebrandt F, et al. The effect of trauma on neutrophil L-selectin expression and sL-selectin serum levels. Shock 2001;15:254-60.

29. Simons RK, Hoyt DB, Winchell RJ, et al. Elevated selectin levels after severe trauma: a marker for sepsis and organ failure and a potential target for immunomodulatory therapy. J Trauma 1997;41:653-61.

30. Seekamp A, van Griensven M, Dhondt E, et al. The effect of anti-L-selectin (aselizumab) in multiply traumatized patients - results of a phase II clinical trial. Crit Care Med 2004;32:2021-8.

31. Oberholzer A, Keel M, Zellweger R, et al. Incidence of septic complications and multiple organ failure in severely injured patients is sex specific. J Trauma 2000;48:932-7.

32. Kubes $P$, Jutila $M$, Payne D. Therapeutic potential of inhibiting leukocyte rolling in ischemia/reperfusion.J Clin Invest 1995;95:2510-9.

33. Tsang YT, Neelamegham S, Hu Y, et al. Synergy between L-selectin signaling and chemotactic activation during neutrophil adhesion and transmigration. J Immunol 1997;159:4566-77.

34. McIntyre TM, Modur V, Prescott SM, et al. Molecular mechanisms of early inflammation. Thromb Haemost 1997;77:302-9.

\section{Address of Correspondence}

Andreas Seekamp, MD, FACS

Klinik für Unfallchirurgie

Universitätsklinikum Schleswig-Holstein

Campus Kiel

Arnold-Heller-Straße 7

24105 Kiel

Germany

Phone (+49/431) 597-4351, Fax -4107

e-mail: andreas.seekamp@unfchir.uni-kiel.de 